\title{
SYMPOSIUM
}

\section{Affective response to eye contact and face recognition ability in children with ASD}

\author{
ROBERT M. JOSEPH, KELLY EHRMAN, REBECCA MCNALLY, AND BRANDON KEEHN \\ Department of Anatomy and Neurobiology, Boston University School of Medicine, Boston, Massachusetts \\ (Received December 19, 2007; Final Revision July 18, 2008; Accepted July 21, 2008)
}

\begin{abstract}
This study tested the hypothesis that affective arousal in response to eye contact is negatively associated with face identification skills in children with autism spectrum disorder (ASD). Participants were 20 children and adolescents with ASD and 20 age- and IQ-matched typically developing (TD) children. Skin conductance response (SCR), a psychophysiological measure of autonomic arousal, was collected while participants viewed faces with gaze directed toward them and faces with gaze averted away from them. Participants also completed an independent match-to-sample face recognition test. Children with ASD exhibited significantly larger SCRs than TD children to faces with direct and averted gaze. There were no differences between SCRs to direct gaze and averted gaze in either group. Children with ASD exhibited a marginally significant decrease in face recognition accuracy relative to TD children, particularly when face recognition depended on the eye region of the face. Face recognition accuracy among children with ASD was negatively correlated with the amplitude of SCRs to direct gaze but not to averted gaze. There was no association between face recognition accuracy and SCRs to gaze in the TD group. These findings suggest that autonomic reactivity to eye contact may interfere with face identity processing in some children with ASD. (JINS, 2008, 14, 947-955.)
\end{abstract}

Keywords: Autistic disorder, Social interaction, Emotion, Arousal, Psychophysiology, Skin electric conductance

\section{INTRODUCTION}

Autism spectrum disorder (ASD) is characterized by qualitative impairments in interpersonal communication and reciprocal social interaction (American Psychiatric Association, 1994). Among these impairments, a deficit in the use of eye contact for social-communicative purposes is one of the most striking and universal features of ASD. There is abundant evidence that individuals with ASD look significantly less to other people's faces and eyes than do typically developing individuals (Hutt \& Ounsted, 1966; Joseph \& Tager-Flusberg, 1997; Kasari et al., 1993; Klin et al., 2002; Pelphrey et al., 2002; Phillips et al., 1992; Volkmar \& Mayes, 1990). Decreased eye contact is already apparent at or before 1 year of age in preschool children subsequently diagnosed with ASD and in infants who carry a familial risk

Correspondence and reprint requests to: Robert M. Joseph, Department of Anatomy and Neurobiology, Boston University School of Medicine, 715 Albany St., L-814, Boston, MA 02118. E-mail: rmjoseph@bu.edu for ASD (Merin et al., 2007; Osterling \& Dawson, 1994; Osterling et al., 2002; Yirmiya \& Ozonoff, 2007). Individuals with ASD are particularly impaired in using eye contact to initiate and regulate social-communicative interactions (Mirenda et al., 1983; Mundy et al., 1986; Swettenham et al., 1998), suggesting that eye contact anomalies have special etiological significance in the development of the core symptoms of ASD.

The causes of reduced eye contact in ASD are not well understood. One plausible explanation is that individuals with ASD do not attend to other people's eyes because of an indifference borne of social-perceptual impairments that render gaze cues uninformative to them. Consistent with this view is evidence that individuals with ASD are impaired at inferring intentions and mental states conveyed through other people's eyes (Baron-Cohen et al., 1995, 1997a,b; but see Back et al., 2007). Using fMRI, Pelphrey et al. (2005) investigated activation of the superior temporal sulcus (STS), the cortical region most associated with gaze processing, in response to goal-directed shifts of gaze in individuals with 
autism. Participants viewed a virtual actor who on alternate trials either gazed toward an object that appeared on the periphery or, instead, looked away from the object toward empty space. Pelphrey et al. found that individuals with autism, in contrast to controls, did not differ in their level of STS activation between instances when the actor's gaze successfully located the object and when it did not, suggesting that they have a diminished perception of gaze as intentional and goal-directed.

An alternative explanation for reduced eye contact in ASD is that gaze behavior anomalies in ASD arise from an aversion to looking at other people's faces and eyes resulting from heightened autonomic response to salient social stimuli (Hutt \& Ounsted, 1966). Level of autonomic arousal is normally mediated not only by the hypothalamus, but by the amygdala and other social-affective brain circuitry, including the anterior cingulate and prefrontal cortex (Davidson \& Irwin, 1999), and is thought to play an important role in the regulation of social, emotional, and motivational behavior (Damasio, 1999). A widely used measure of individual differences and phasic changes in autonomic arousal is skin conductance response. A skin conductance response (SCR) is a momentary change in the electrical conductance of the skin produced by activity of the eccrine sweat glands, which are under the control of the autonomic nervous system. In a recent study, Kylliainen and Hietanen (2006) measured SCRs in 12 high-ability, school-age children with autism who viewed 6-s moving images of 6 male and 6 female faces, all in full-frontal orientation, half with direct gaze and half with gaze averted to the left or right. In each film clip, the face gradually increased in visual angle, giving the impression that the face was looming toward viewer. Kylliainen and Hietanen found that children with autism generated larger SCRs to faces with direct gaze than to faces with averted gaze, whereas the control group responded equivalently to the two gaze conditions.

Experimental research has demonstrated that individuals with ASD are not only impaired in processing gaze cues, but that they have deficiencies in other aspects of face processing, such as learning and recognizing novel faces (see Sasson, 2006, for a review). Although laboratory evidence of ASD deficits in face identity learning and recognition have often been argued to reflect a failure of configural processing mechanisms normally essential to face encoding (Behrmann et al., 2007; Boucher \& Lewis, 1992; Davies et al., 1994; but see Joseph \& Tanaka, 2003; Lahaie et al., 2006; Rouse et al., 2004), other evidence suggests that autistic face processing impairments derive specifically from abnormalities in encoding information from the eye region of the face, the area of the face that is normally most informative for face recognition (Goldstein \& Mackenberg, 1966; McKelvie, 1976; Sergent, 1984; Tanaka \& Farah, 1993). For example, Joseph and Tanaka (2003) found evidence of intact holistic face recognition processes in children with autism, but only when face recognition depended on processing information from the mouth area of the face and not when it depended on the eyes. These findings were consis- tent with those from a much earlier study by Langdell (1978), who reported that children with autism were better at recognizing photos of their peers based on isolated information from the mouth region than from the eye region. In a similar vein, using the "Bubbles" method (Gosselin \& Schyns, 2001) to assess the degree to which different regions of the face were used to identify facial expressions, Spezio et al. (2006) reported that adults with autism were significantly less reliant on the eye region and more reliant on the mouth region in their emotion judgments than nonautistic adults. Together, these studies suggest that autistic deficits in face processing derive from abnormalities in processing information from the eyes rather than from an absolute deficit in holistic face processing.

In the present study, we sought to test the hypothesis that psychophysiological arousal in response to looking at other people's faces and particularly their eyes may be associated with deficits in encoding and recognizing novel faces in children and adolescents with ASD. To do so, we assessed children's autonomic response, as measured by SCR, to faces with gaze directed toward the participant and, as a control condition, to faces with gaze directed away from the participant. We assessed children's face recognition abilities independently of their SCRs to direct and averted gaze using a separate two-alternative, forced-choice, match-to-sample procedure in which the test faces (all with direct gaze) differed on one facial feature, either the eyes or the mouth. We predicted that face recognition performance, particularly on trials in which recognition depended on encoding information from the eye area of the face, would be negatively associated with the amplitude of SCRs to faces with direct gaze but unrelated to the amplitude of SCRs to faces with averted gaze in children with ASD. In contrast, we expected that face recognition performance in an age- and IQ-matched comparison group of typically developing (TD) children would be unrelated to SCRs to faces, regardless of gaze direction. An additional aim was to examine whether the findings of Kylliainen and Hietanen (2006), who used dynamic stimuli to assess psychophysiological arousal to direct versus averted gaze in children with ASD, would hold for static gaze stimuli that did not appear to approach the observer. Based on their findings, we predicted that children with ASD would exhibit larger SCRs to faces with direct gaze than to faces with averted gaze, whereas TD children would not differ in their response to the two gaze conditions.

\section{METHODS}

\section{Participants}

Participants were 20 school-age children with ASD (18 males), all of whom were judged to meet DSM-IV (American Psychiatric Association, 1994) criteria for autism or PDDNOS by an expert clinician, and a comparison group of 20 typically developing (TD) children (13 males). ASD diagnoses were confirmed using the Autism Diagnostic 
Table 1. Participant characteristics

\begin{tabular}{lcc}
\hline \hline & ASD $(n=20)$ & TD $(n=20)$ \\
& $M(S D)$, Range & $M(S D)$, Range \\
\hline Age & $12 ; 6(2 ; 0), 9 ; 1-16 ; 1$ & $11 ; 11(1 ; 9), 8 ; 7-15 ; 6$ \\
Verbal IQ & $98(20), 51-134$ & $102(16), 73-130$ \\
Nonverbal IQ & $107(20), 71-152$ & $101(16), 68-138$ \\
\hline \hline
\end{tabular}

Interview-Revised (ADI-R; Rutter et al., 2003), an experimenter-administered, parent interview based primarily on behaviors reported for the 4- to 5-year age period, and the Autism Diagnostic Observation Schedule (ADOS; Lord et al., 1999), an experimenter-administered, interactive observation measure which provides direct behavioral ratings of autism symptoms in the social and communication domains. All children in the ASD group met criteria for an ADI-R classification of autism, with the exception of two children who were one point below the diagnostic threshold in the repetitive behavior domain. On the ADOS, 14 children met classification criteria for autism, 4 met for a less severe classification of autism spectrum disorder, and 2 met for an ADOS classification of autism in the social domain, but were below threshold in the communication domain. The latter two children met full criteria for autism on the ADI-R, and were therefore included in the sample. Children with autism-related medical conditions (e.g., Fragile-X syndrome, tuberous sclerosis) were not included in this study. All comparison group participants had no reported history of autism and were confirmed to be free of autism-related symptoms and of any other neurological or psychiatric conditions via parent report and expert clinical observation. One-way between-group analyses of variance (ANOVAS) confirmed that the autism and comparison groups were matched on age, $F(1,38)=$ $0.7 ; p=.41$, and on verbal IQ, $F(1,38)=0.4 ; p=.55$, and nonverbal IQ, $F(1,38)=1.1 ; p=.30$, as measured by the Differential Ability Scales (DAS; Elliott, 1990). See Table 1. Written informed consent or verbal assent was obtained from all participants and their parents. This study was approved by the Institutional Review Board of the Boston University Medical Center.

\section{Experimental Measures}

The following two measures were administered to each participant. Order of administration was counterbalanced across participants.

\section{SCRs to direct and averted gaze}

Face stimuli were made from high-resolution, grayscale digital photos of six male and six female faces. Each face was photographed with the same background and under the same lighting conditions in four poses in which two directions of gaze (directly toward the camera/observer or averted laterally by $30^{\circ}$ ) were crossed with two head orientations (oriented frontally toward the camera/observer or turned in profile orientation by $30^{\circ}$ ), yielding four stimulus types: head frontal/gaze direct (frontal-direct); head profile/gaze direct (profile-direct); head frontal/gaze averted (frontalaverted); and head profile/gaze averted (profile-averted) (see Fig. 1). Adobe Photoshop 7.0 was used to adjust for slight differences in the luminance and contrast in the images, and to position each face such that the eyes always appeared in the same location of the frame. Images were cropped rectangularly to approximately $15 \mathrm{~cm}$ in width and $22 \mathrm{~cm}$ in height, varying somewhat according to head size and shape. The horizontal flip function of Photoshop was used to make identical left-right mirror images of the faces that were in profile or with averted gaze.

The experiment included all 12 faces, each of which was presented two times with each gaze direction (direct and averted) in the same head orientation (either frontal or profile), for a total of 24 trials completely crossed for sex, head orientation, gaze direction, and the side to which the head or gaze was directed (left or right). The faces were counterbalanced for identity across head orientation, such that half the participants saw six faces (three males, three females)
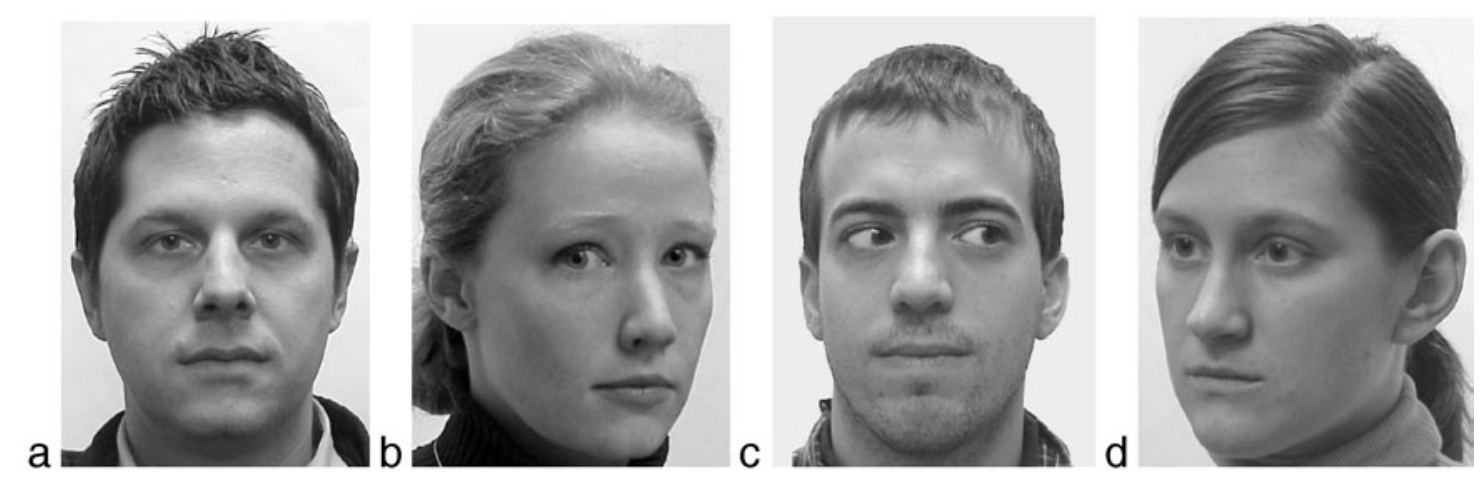

Fig. 1. Samples of the four types of gaze stimuli crossing head orientation with gaze direction: (a) frontal-direct; (b) profile-direct; (c) frontal-averted; (d) profile-averted. 

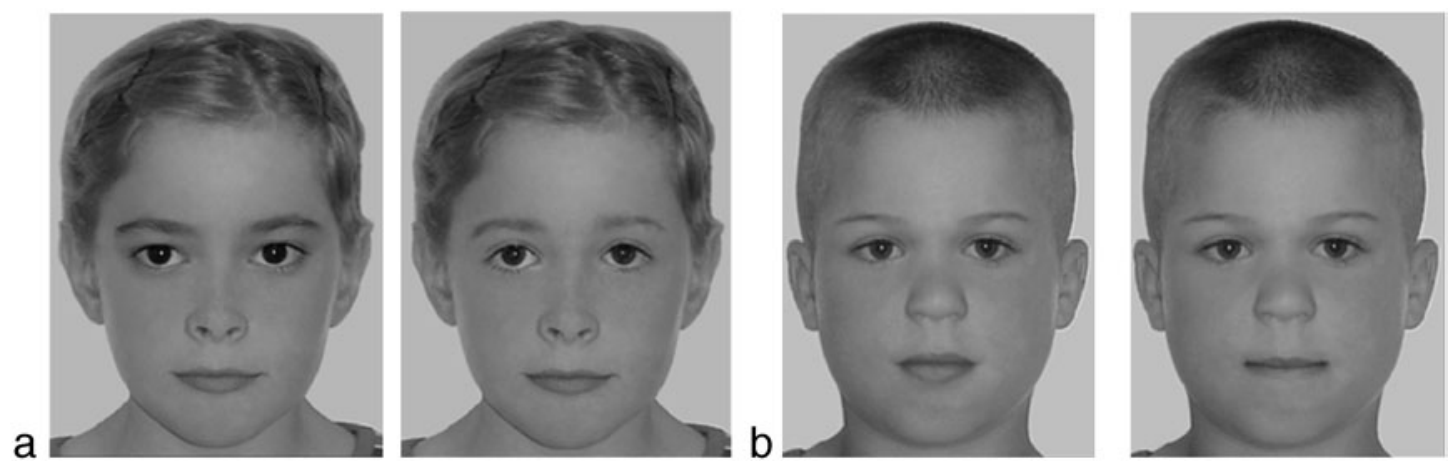

Fig. 2. Samples of target and foil faces for (a) eyes condition and (b) mouth condition of the face recognition test.

in frontal orientation and the other six faces in profile orientation, and vice versa. The faces were presented in one of four pseudorandom orders with SuperLab Pro 2.0 software on an IBM-compatible, Pentium III processor computer with a 17-inch CRT monitor. Each face was presented for $4 \mathrm{~s}$ with a variable 5- to 7-s interstimulus interval from offset to onset of the next face. Participants were seated approximately $57 \mathrm{~cm}$ from the monitor, and were asked to look at the faces and to try to remember them. Following the main experiment, participants viewed each of the 12 faces paired with a foil face of the same gender not previously presented and were asked to indicate via a two-choice button box response which one they had seen.

SCRs were acquired with a James Long bio-amplifier and PHY General Physiology System and were analyzed using James Long SCOR2 software. Silver/silver chloride electrodes filled with a biopotential contact medium were placed on the medial phalanges of the index and middle fingers of the participant's nondominant hand. Participants spent several minutes acclimating to wearing the electrodes before the experiment. All SCR data were prescreened to eliminate artifactual responses related to hand and other body movements. An SCR was defined as the maximum rise in amplitude from basepoint, measured in microsiemens $(\mu \mathrm{S})$, occurring during an 8-s window starting $1 \mathrm{~s}$ after stimulus onset, with the additional requirement that the rise in amplitude had to begin (although not necessarily reach peak) within the first $4 \mathrm{~s}$ of that window to help ensure that it was elicited by the stimulus (Levinson \& Edelberg, 1985). To correct for individual differences in tonic skin conductance level (SCL), which typically ranges from 2 to $20 \mu \mathrm{S}$, all SCRs were divided by the participant's average baseline SCL. Baseline SCL was averaged from $2 \mathrm{~min}$ of data collected at the end of the acclimation period and immediately before the testing procedure. This correction served to reduce error variance related to extraneous factors (e.g., skin thickness, sweat gland density, general response to the laboratory environment) and thereby increase power to detect differences in the psychological variables under consideration (Dawson et al., 2000).

\section{Face recognition}

The face stimuli and testing procedure were the same as in Joseph and Tanaka (2003) except that only recognition of whole faces in upright orientation was tested. On each trial, participants viewed a high-resolution, grayscale digital image of a target face for $3.5 \mathrm{~s}$ followed by an immediate twochoice, match-to-sample recognition test in which the target face was presented alongside a foil face that differed from the target on either the eyes or mouth. See Figure 2. The two faces remained on the screen for $8 \mathrm{~s}$ or until a response was made. Each face stimulus (six boys, six girls) measured approximately $12 \mathrm{~cm}$ in height and $10 \mathrm{~cm}$ in width and was presented two times, once in the eye condition and once in the mouth condition, with participants seated approximately $57 \mathrm{~cm}$ from the monitor. A total of 24 trials were presented in one of two pseudorandom orders counterbalanced across participants. The experiment was presented using SuperLab Pro 2.0 software on a Pentium III PC with a 17-inch CRT monitor and test responses were registered using a Cedrus RB-410 button box.

\section{RESULTS}

\section{SCRs to Direct and Averted Gaze}

Participants in both groups performed at ceiling levels of accuracy in the posttest assessing memory of the face stimuli used in the SCR procedure, indicating that they were attending adequately. The ceiling performance of the ASD group on this posttest was not surprising given that foil and target faces were easy to discriminate (in contrast to the faces in the main recognition test) as long as participants were attending to them. Mean, standard deviations, and ranges for baseline SCL and for SCRs (raw and corrected for baseline SCL) to direct and averted gaze stimuli are reported in Table 2. As in Kylliainen and Hietanen (2006), mean SCRs were calculated based on all trials including those with a zero response. The ASD and TD groups did not differ in mean SCL, $F(1,38)=0.0$. The two groups exhibited similarly wide ranges of SCL which were comparable 
Table 2. Mean amplitude (raw and corrected for SCL) of SCRs to direct and averted gaze

\begin{tabular}{lcc}
\hline \hline & ASD $(n=20)$ & TD $(n=20)$ \\
& $M(S D)$, Range & $M(S D)$, Range \\
\hline Baseline SCL & $11.0(5.4), 2.4-25.0$ & $10.9(7.0), 3.5-24.9$ \\
Mean raw amplitude & & \\
$\quad$ Direct gaze & $.067(.08), 0-.32$ & $.053(.09), 0-.41$ \\
$\quad$ Averted gaze & $.076(.11), 0-.42$ & $.050(.11), 0-.40$ \\
Mean corrected amplitude & & \\
$\quad$ Direct gaze & $.0060(.005), 0-.015$ & $.0034(.005), 0-.016$ \\
$\quad$ Averted gaze & $.0063(.007), 0-.023$ & $.0033(.006), 0-.020$ \\
\hline \hline
\end{tabular}

in the degree of variance (Levene statistic $=1.6 ; p=.22$ ). Both the raw and corrected SCR distributions showed significant positive skew due to relatively large responses by a minority of individuals in each group. Neither square root nor logarithmic transformations (Tabachnick \& Fidell, 2001) improved the distributions sufficiently for parametric statistical analyses, and therefore nonparametric statistics were used. All statistical analyses were conducted on the SCR data corrected for baseline SCL.

Mann-Whitney $U$-tests were conducted to assess group differences in the mean amplitude of SCRs in the direct and averted gaze conditions. The ASD group exhibited a significantly higher mean response than the TD group to both faces with direct gaze, $z=2.2, p<.05$, and faces with averted gaze, $z=2.1, p<.05$. Because there were more females in the TD group than in the ASD group, comparisons of mean SCR amplitude to direct and averted gaze by participant sex were conducted for the TD group to ensure that sex-based differences in SCRs did not account for the group differences found. These analyses revealed no difference between TD males and females to direct gaze, $z=0.3$, n.s., or to averted gaze, $z=0.9$, n.s.

In addition to between-group comparisons, within-group comparisons of the mean amplitude of SCRs to direct as compared to averted gaze stimuli were conducted using Wilcoxon signed ranked tests. These tests showed no difference in amplitude of response between the direct and averted gaze conditions in the ASD group, $z=0.3$, n.s., and in the TD group, $z=0.1$, n.s.

\section{Face Recognition}

A mixed-model ANOVA with the factors group and facial feature (eyes, mouth) was conducted on face recognition accuracy scores. There was a marginally significant trend toward decreased face recognition accuracy in the ASD group relative to the TD group, $F(1,38)=2.8 ; p<.10$. Based on the findings of Joseph and Tanaka (2003), planned post hoc group comparisons were conducted to assess feature-based differences between groups. These analyses revealed no group difference in the mouth condition (ASD: $M=8.9$, $S D=1.4$; TD: $M=9.4, S D=1.6 ; F(1,38)=0.9$, n.s. $)$, but a marginally significant difference in the eyes condition
(ASD: $M=8.9, S D=2.3$; TD: $M=10.1, S D=1.8$; $F(1,38)=2.8, p<.10)$.

\section{SCRs to Gaze and Face Recognition Accuracy}

Spearman rho correlations were conducted to assess the relationships between autonomic arousal in response to direct and averted gaze and face recognition abilities in each group. Face recognition accuracy in the ASD group was negatively correlated with mean amplitude of SCRs to faces with direct gaze, $r(18)=-.79 ; p<.001$, but was unrelated to mean amplitude of SCRs to faces with averted gaze, $r(18)=-.28$. A Williams T2 test (Steiger, 1980) to assess differences between nonindependent correlations showed that the correlation between face recognition and amplitude of SCRs to direct gaze was of significantly larger magnitude than that between face recognition and amplitude of SCRs to averted gaze stimuli, $t(17)=5.3 ; p<.001$. Mean amplitude of SCRs to direct gaze faces was negatively correlated both with eye-based face recognition accuracy, $r(18)=-.77 ; p<.001$, and mouth-based face recognition accuracy, $r(18)=-.58 ; p<.01$, in the ASD group. The difference in magnitude between these two correlations, $t(17)=1.3 ; p<.10$, approached statistical significance.

Among TD participants, there was no association between face recognition accuracy and mean amplitude of SCRs to direct gaze faces, $r(18)=-.12$, or SCRs to averted gaze, $r(18)=.07$. Figure 3 illustrates the correlation between amplitude of SCRs to direct gaze and face recognition scores in ASD and TD participants. Additional analyses showed similarly small correlations between eye-based face recog-

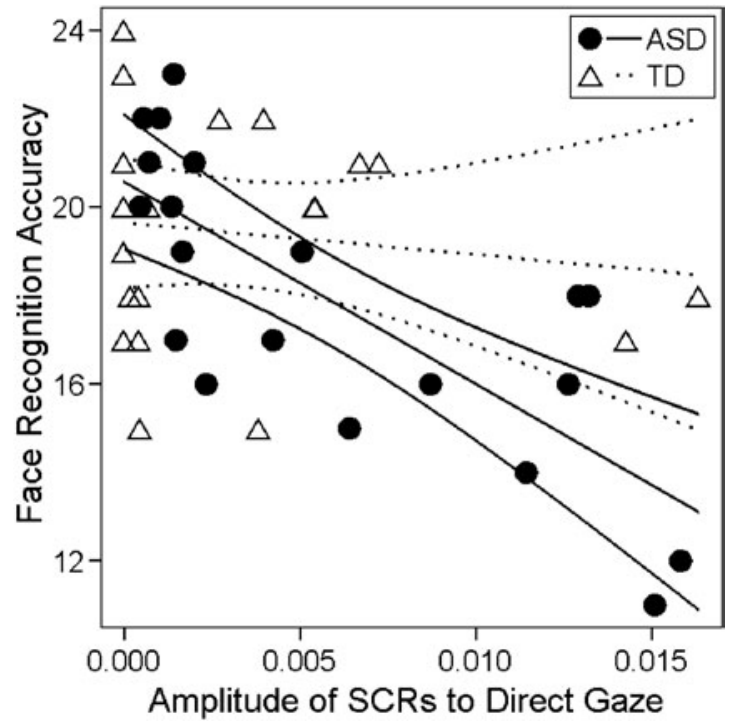

Fig. 3. Correlation between amplitude of SCRs to faces with direct gaze (corrected for baseline SCL) and face recognition accuracy score with $95 \%$ confidence intervals. 
nition accuracy and amplitude of SCRs to direct gaze, $r(18)=$ -.17 , and averted gaze, $r(18)=-.01$, and between mouthbased face recognition accuracy and amplitude of SCRs to direct gaze, $r(18)=-.05$, and averted gaze, $r(18)=-.10$, in the TD group.

\section{DISCUSSION}

We assessed whether individual differences in autonomic arousal in response to eye contact were related to differences in the ability to encode and recognize faces in children with ASD. As predicted, we found that face recognition accuracy among children with ASD correlated strongly and negatively with the amplitude of SCRs to direct gaze faces, but was unrelated to the amplitude of SCRs to averted gaze faces. Thus, although children with ASD evidenced similarly heightened levels of autonomic response to direct gaze and averted gaze, only their response to the former had explanatory value with regard to their face recognition skills. Furthermore, autonomic response to direct gaze was more strongly correlated with face recognition based on the eye region than on the mouth region of the face, but only marginally so. Although preliminary and in need of further investigation, the significant associations with mouth-based as well as eye-based face recognition suggests that autonomic reactivity to direct gaze, in so far as it represents an emotionally aversive response to eye contact, may affect processing not only of the eye region but of the face in general, at least in some individuals with ASD.

In contrast to the ASD group, there was no association between face recognition performance and SCRs to gaze stimuli in the TD group. Given that the mean amplitude of the SCRs of the TD group was roughly half that of the ASD group in both the direct and averted gaze conditions once corrected for baseline SCL, it is worth noting that the lack of a similar correlation between autonomic response and face recognition in the TD group does not appear to result from a restricted range of autonomic response. As can be seen in Table 2, the range of response was comparable between groups. In addition, inspection of Figure 3 shows that approximately half of both groups responded minimally to the direct gaze stimuli while the remaining participants in both groups showed a fairly wide range of response. The same line of argument applies to the lack of association between face recognition accuracy and autonomic response to averted gaze faces in the ASD and TD groups, as the SDs and ranges in Table 2 show.

Contrary to our prediction, children and adolescents with ASD exhibited larger SCRs than the TD comparison group not only to faces with direct gaze but also to faces with averted gaze, and their responses to direct gaze faces were no larger than their responses to averted gaze faces. Thus, there was no evidence of increased autonomic response specifically to eye contact; rather the ASD group exhibited heightened autonomic arousal relative to the comparison group to all face stimuli, regardless of gaze. These findings differed from those of Kylliainen and Hietanen (2006), who found that school-age children with autism exhibited significantly larger SCRs to direct than to averted gaze faces, in contrast to TD children who showed no difference in their response to these two conditions.

Methodological differences between the two studies may help to explain our different findings. For example, whereas Kylliainen and Hietanen (2006) used dynamic stimuli designed to give viewers the impression of a person's face looming over them, we used static stimuli, which eliminated the element of social approach that was effected with Kylliainen and Hietanen's stimuli. Furthermore, their participants were asked to determine the gaze direction of the face stimulus while SCRs were being acquired, which required them to look directly at the eyes. In contrast, we attempted to motivate our participants to attend to and examine the face stimuli simply by asking them to "try to remember them." These methodological differences are perhaps reflected in the fact that Kylliainen and Hietanen reported mean SCRs several times the magnitude of the responses elicited in our study (although baseline SCL data in our study were in the expected range ruling out the possibility of equipment failure). This raises the possibility that the stimuli and procedure we used presented a relatively subtle social press to our participants with ASD, one that was not sufficiently robust to detect a differential response to direct as compared to averted gaze.

We have considered two explanations for the markedly reduced eye contact that is characteristic of ASD. One is that a perceptual or higher cognitive impairment in reading intentional states and social cues communicated through people's eyes and shifts of gaze may result in an indifference or obliviousness to this normally crucial medium for human nonverbal communication. Alternatively, engaging the gaze of another individual may be overly stimulating to individuals with autism, possibly causing them to avoid eye contact so as to regulate affective arousal. We have provided partial support for the latter explanation, showing that emotional arousal in response to direct gaze was negatively associated with face recognition performance in a sample of children and adolescents with ASD, suggesting that psychophysiological aversion to eye contact negatively affects face encoding and identification skills in some individuals with ASD. However, it is important to emphasize that the specific negative relationship we identified between autonomic arousal in response to eye contact and face processing skills occurred in the broader context of indications of a generalized heightened autonomic response to faces regardless of gaze direction, relative to TD children. Thus, on the basis of the current findings, we cannot claim any special status for eye contact aversion in autistic social deficits, but only in the more particular case of face identity processing, in which information from the eyes plays a critical role. Moreover, our main finding of a relationship between affective arousal and face recognition skills was correlational in nature and does not, with regard to the alternatives we have considered, establish causal primacy of affective dysregulation in the social information processing 
deficits and related symptomatology of ASD. In fact, impaired social perception and affective dysregulation may be mutually reinforcing. For example, unusual autonomic reactivity to eye contact could conceivably emerge as an acquired anxiety response in individuals who are regularly pressed to respond to gaze cues they do not understand. Thus, although our findings and those of others suggest that heightened affective reactivity can play a role in autistic social deficits, whether it is more cause or consequence in the disorder awaits future research.

Growing evidence that ASD is not only extremely heterogeneous in its phenotypic expression but also in its genetic etiology (Courchesne et al., 1999; Geschwind, 2007; Gupta $\&$ State, 2007) suggests another possibility, that increased susceptibility to social anxiety and/or affective dysregulation may be one of many risk factors contributing to the development of the highly complex behavioral phenotype we refer to as autism or ASD. In other words, what we have described in terms of social indifference versus social aversion (or anxiety) may represent partially dissociable subtypes with different developmental courses. The possibility that primary deficits in social-affective regulation could distinguish a subtype of ASD is suggested by findings that co-morbid symptoms of mood and anxiety disorders are over-represented in individuals with autism and in their immediate and extended families (Lainhart, 1999; Leyfer et al., 2006; Piven, 1999). This possibility is also consistent with the fact that only approximately half of the ASD participants in the present study exhibited evidence of heightened autonomic arousal in response to the face stimuli presented.

Given that autism is fundamentally a disorder of development, longitudinal research is required to provide a full understanding of the relationship between disturbances in social-affective and social-perceptual functioning in individuals with ASD. In particular, prospective longitudinal study of young children with or at-risk for ASD will help to determine the primary or secondary nature of socialemotional hyper-reactivity in autism and whether heightened autonomic reactivity to social stimuli might segregate into an ASD subtype. Research on autonomic reactivity and social-affective functioning in early childhood will be facilitated by rapidly improving technology for in vivo eyetracking and psychophysiological monitoring that will allow more controlled laboratory experimentation, typically involving computer-presented stimuli, to be complemented by assessments of children in closer approximations of reallife social environments. In addition, although an abnormal pattern of amygdala development in ASD in which early overgrowth is followed by later atrophy is becoming welldocumented in the autism literature (Sparks et al., 2002; Schumann et al., 2004; Schumann \& Amaral, 2006), and the effects of hyper-reactivity to social stress have been at least theoretically implicated in this anomalous pattern of amygdala development (Nacewicz et al., 2006; Schumann \& Amaral, 2006), further research is necessary to explore how structural and functional abnormalities of the amyg- dala may be related to developmental neuropathology of other components of social-affective neural circuitry, including the prefrontal cortices.

\section{ACKNOWLEDGMENTS}

This research was funded by NIDCD grant U19 DC 03610 (Project 1), which is part of the NICHD/NIDCD Collaborative Programs of Excellence in Autism, and by NIMH grant K01 MH 073944. We thank James Long for his technical assistance in SCR data analysis and the anonymous reviewers and symposium editors for their helpful comments. We are especially grateful to the children and families who generously participated in this study.

\section{REFERENCES}

American Psychiatric Association. (1994). Diagnostic and Statistical Manual of Mental Disorders (DSM-IV, 4th ed.). Washington, DC: American Psychiatric Association Press.

Back, E., Ropar, D., \& Mitchell, P. (2007). Do the eyes have it? Inferring mental states from animated faces in autism. Child Development, 78, 397-411.

Baron-Cohen, S., Baldwin, D.A., Crowson, M. (1997a). Do children with autism use the speaker's direction of gaze strategy to crack the code of language? Child Development, 68, 48-57.

Baron-Cohen, S., Campbell, R., Karmiloff-Smith, A., Grant, J., \& Walker, J. (1995). Are children with autism blind to the mentalistic significance of the eyes? British Journal of Developmental Psychology, 13, 379-398.

Baron-Cohen, S., Wheelwright, S., \& Jolliffe, T. (1997b). Is there a "language of the eyes"? Evidence from normal adults and adults with autism or Asperger syndrome. Visual Cognition, 4, 311-331.

Behrmann, M., Avidan, G., Leonard, G.L., Kimchi, R., Luna, B., Humphreys, K., \& Minshew, N. (2007). Configural processing in autism and its relationship to face processing. Neuropsychologia, 44, 110-129.

Boucher, J. \& Lewis, V. (1992). Unfamiliar face recognition in relatively able autistic children. Journal of Child Psychology and Psychiatry, 33, 843-859.

Courchesne, E., Yeung-Courchesne, R., \& Pierce, K. (1999). Biological and behavioral heterogeneity in autism: Roles of pleiotropy and epigenesis. In S.H. Broman \& J.M. Fletcher (Eds.), The Changing Nervous System: Neurobehavioral Consequences of Early Brain Disorders (pp. 292-338). New York: Oxford University Press.

Damasio, A.R. (1999). The Feeling of What Happens: Body and Emotion in the Making of Consciousness. New York: Harcourt Brace.

Davidson, R.J. \& Irwin, W. (1999). The functional neuroanatomy of emotion and affective style. Trends in Cognitive Sciences, 3 , $11-21$.

Davies, S., Bishop, D., Manstead, A.S., \& Tantam, D. (1994). Face perception in children with autism and Asperger's syndrome. Journal of Child Psychology and Psychiatry, 35, 1033-1057.

Dawson, M.E., Schell, A.M., \& Filion, D.L. (2000). The electrodermal system. In J.T. Cacioppo, L.G. Tassinary, \& G.G. Bernston (Eds.), Handbook of Psychophysiology (2nd ed.). New York: Cambridge University Press.

Elliott, C.D. (1990). Differential Ability Scales: Introductory and Technical Handbook. New York: The Psychological Corporation. 
Geschwind, D. (2007). Autism: Searching for coherence. Biological Psychiatry, 62, 949-950.

Goldstein, A.G. \& Mackenberg, E. (1966). Recognition of human faces from isolated facial features. Psychonomic Science, 6 , $149-150$

Gosselin, F. \& Schyns, P.G. (2001). Bubbles: A technique to reveal the use of information in recognition tasks. Vision Research, $41,2261-2271$.

Gupta, A.R. \& State, M.W. (2007). Recent advances in the genetics of autism. Biological Psychiatry, 61, 429-437.

Hutt, C. \& Ounsted, C. (1966). The biological significance of gaze aversion with particular reference to the syndrome of infantile autism. Behavioral Science, 11, 346-356.

Joseph, R.M. \& Tager-Flusberg, H. (1997). An investigation of attention and affect in children with autism and Down syndrome. Journal of Autism and Developmental Disorders, 4, 385-396.

Joseph, R.M. \& Tanaka, J. (2003). Holistic and part-based face recognition in children with autism. Journal of Child Psychology and Psychiatry, 44, 529-542.

Kasari, C., Sigman, M., \& Yirmiya, N. (1993). Focused and social attention of autistic children in interactions with familiar and unfamiliar adults: A comparison of autistic, mentally retarded, and normal children. Development and Psychopathology, 5, 403-414.

Klin, A., Jones, W., Schultz, R., Volkmar, F., \& Cohen, D.J. (2002). Visual fixation patterns during viewing of naturalistic social situations as predictors of social competence in individuals with autism. Archives of General Psychiatry, 59, 809816

Kylliainen, A. \& Hietanen, J.K. (2006). Skin conductance responses to another person's gaze in children with autism. Journal of Autism and Developmental Disorders, 36, 517-525.

Lahaie, A., Mottron, L., Arguin, M., Berthiaume, C., Jemel, B., \& Saumier, D. (2006). Face perception in high-functioning autistic adults: Evidence for superior processing of face parts, not for a configural face processing deficit. Neuropsychology, 20, $30-41$.

Lainhart, J.E. (1999). Psychiatric problems in individuals with autism, their parents and siblings. International Review of Psychiatry, 11, 278-298.

Langdell, T. (1978). Recognition of faces: An approach to the study of autism. Journal of Child Psychology and Psychiatry, 19, 255-268.

Levinson, D.F. \& Edelberg, R. (1985). Scoring criteria for response latency and habituation in electrodermal research: A critique. Psychophysiology, 22, 417-426.

Leyfer, O.T., Folstein, S.E., Bacalman, S., Davis, N.O., Dinh, E., Morgan, J., Tager-Flusberg, H., \& Lainhart, J.E. (2006). Comorbid psychiatric disorders in children with autism: Interview development and rates of disorders. Journal of Autism and Developmental Disorders, 36, 849-861.

Lord, C., Rutter, M., DiLavore, P.C., \& Risi, S. (1999). Autism Diagnostic Observation Schedule-WPS (ADOS-WPS). Los Angeles, CA: Western Psychological Services.

McKelvie, S.J. (1976). The role of eyes and mouth in the memory of a face. American Journal of Psychology, 89, 311-323.

Merin, N., Young, G.S., Ozonoff, S., \& Rogers, S.J. (2007). Visual fixation patterns during reciprocal social interaction distinguish a subgroup of 6-month-old infants at-risk for autism from comparison infants. Journal of Autism and Developmental Disorders, 37, 108-121.
Mirenda, P., Donnellan, A., \& Yoder, D. (1983). Gaze behavior: A new look at an old problem. Journal of Autism and Developmental Disorders, 13, 397-409.

Mundy, P., Sigman, M., Ungerer, J., \& Sherman, T. (1986). Defining the social deficits in autism: The contribution of nonverbal communication measures. Journal of Child Psychology and Psychiatry, 27, 657-669.

Nacewicz, B.M., Dalton, K.M., Johnstone, T., Long, M., McAuliff, E.M., Oakes, T.R., Alexander, A.L., \& Davidson, R.J. (2006). Amygdala volume and nonverbal social impairment in adolescent and adult males with autism. Archives of General Psychiatry, 63, 1417-1428.

Osterling, J. \& Dawson, G. (1994). Early recognition of children with autism: A study of first birthday home videotapes. Journal of Autism and Developmental Disorders, 24, 247-257.

Osterling, J.A., Dawson, G., \& Munson, J.A. (2002). Early recognition of 1-year-old infants with autism spectrum disorder versus mental retardation. Development and Psychopathology, 14 , 239-251.

Pelphrey, K.A., Morris, J.P., \& McCarthy, G. (2005). Neural basis of eye gaze processing deficits in autism. Brain, 128, 1038-1048.

Pelphrey, K.A., Sasson, N.J., Reznick, J.S., Paul, G., Goldman, B.D., \& Piven, J. (2002). Visual scanning of faces in autism. Journal of Autism and Developmental Disorders, 32, 249-261.

Phillips, W., Baron-Cohen, S., \& Rutter, M. (1992). The role of eye contact in the detection of goals: Evidence from normal toddlers and children with autism or mental handicap. Development and Psychopathology, 4, 375-383.

Piven, J. (1999). Genetic liability for autism: The behavioural expression in relatives. International Review of Psychiatry, 11 , 299-308.

Rouse, H., Donnelly, N., Hadwin, J.A., \& Brown, T. (2004). Do children with autism perceive second-order relational features? The case of the Thatcher illusion. Journal of Child Psychology and Psychiatry and Allied Disciplines, 45, 1246-1257.

Rutter, M., Le Couteur, A., \& Lord, C. (2003). Autism Diagnostic Interview-Revised. Los Angeles, CA: Western Psychological Services.

Sasson, N.J. (2006). The development of face processing in autism. Journal of Autism and Developmental Disorders, 36, 381-394.

Schumann, C.M. \& Amaral, D.G. (2006). Stereological analysis of amygdala neuron number in autism. The Journal of Neuroscience, 26, 7674-7679.

Schumann, C.M., Hamstra, J., Goodlin-Jones, B.L., Lotspeich, L.J., Kwon, H., Buonocore, M.H., Lammers, C.R., Reiss, A.L., \& Amaral, D.G. (2004). The amygdala is enlarged in children but not adolescents with autism; the hippocampus is enlarged at all ages. The Journal of Neuroscience, 24, 6392-6401.

Sergent, J. (1984). An investigation into component and configural processes underlying face perception. The British Journal of Psychology, 75, 221-242.

Sparks, B.F., Friedman, S.D., Shaw, D.W., Aylward, E.H., Echelard, D., Artru, A.A., Maravilla, K.R., Giedd, J.N., Munson, J., Dawson, G., \& Dager, S.R. (2002). Brain structural abnormalities in young children with autism spectrum disorder. $\mathrm{Neu}$ rology, 59, 184-192.

Spezio, M.L., Adolphs, R., Hurley, R.S., \& Piven, J. (2006). Abnormal use of facial information in high-functioning autism. Journal of Autism and Developmental Disorders, 37, 929-939 
Steiger, J.H. (1980). Tests for comparing elements of a correlation matrix. Psychological Bulletin, 88, 245-258.

Swettenham, J., Baron-Cohen, S., Charman, T., Cox, A., Baird, G., Drew, A., Rees, L., \& Wheelwright, S. (1998). The frequency and distribution of spontaneous attention shifts between social and non-social stimuli in autistic, typically developing, and non-autistic developmentally delayed infants. Journal of Child Psychology and Psychiatry, 9, 747-753.

Tabachnick, B.G. \& Fidell, L.S. (2001). Using Multivariate Statistics (4th ed.). Needham Heights, MA: Allyn \& Bacon.
Tanaka, J.W. \& Farah, M.J. (1993). Parts and wholes in face recognition. Quarterly Journal of Experimental Psychology, 46A, $225-245$

Volkmar, F.R. \& Mayes, L.C. (1990). Gaze behavior in autism. Development and Psychopathology, 2, 61-69.

Yirmiya, N. \& Ozonoff, S. (2007). The very early autism phenotype. Journal of Autism and Developmental Disorders, 37, 1-11. 